\title{
1 \\ Specification-based testing of concurrent systems
}

\author{
Andreas Ulrich ${ }^{a}$, Hartmut König ${ }^{b}$ \\ ${ }^{a}$ Dept. of Comp. Science, Univ. of Magdeburg, PF 4120, 39016 \\ Magdeburg,Germany; e-mail: ulrich@cs.uni-magdeburg.de \\ ${ }^{b}$ Dept. of Computer Science, BTU Cottbus, PF 101344, 03013 \\ Cottbus, Germany; e-mail: koenig@informatik.tu-cottbus.de
}

\begin{abstract}
The paper addresses the problem of test suite derivation from a formal specification of a distributed concurrent software system given as a collection of labeled transition systems. It presents a new concurrency model, called behavior machine, and its construction algorithm. Further, the paper outlines how test derivation can be based on the new concurrency model in order to derive test suites that still exhibit true concurrency between test events. A toolset is presented to support the generation of concurrent test suites from specifications given in the formal description technique LOTOS. Finally, some comments on requirements for the design of a distributed test architecture are given.
\end{abstract}

\section{Keywords}

Distributed concurrent software systems; conformance testing; test derivation; labeled transition systems; LOTOS; Petri nets.

\section{INTRODUCTION}

Testing is an important means in the development cycle of software. A challenging problem is the derivation of test suites that are able to detect faulty implementations 
of a system firmly. Driven by requirements in testing telecommunication systems, approaches were developed to assist the automatic derivation of test suites [ADL+91] [Fer96]. These approaches are usually based on a finite description of the behavior of the system, mostly the model of a finite state machine, that is also exploited in the verification phase of the system. However, current test derivation approaches only support the derivation of test suites for sequential systems. One reason is that they are faced with computational problems due to state explosion if they resolve specified concurrent behavior in an interleaving sequence of actions of a derived test suite. Furthermore, its execution in a standard black-box test architecture might be not sufficient to assess conformance of truly concurrent systems since the message exchange between components of the concurrent system must be observed and controlled by a tester in order to avoid nondeterministic test runs.

This paper continues work on the use of partial orders for test suite derivation of concurrent systems. It improves the previous work done in [U1Ch95] and also other known work on this subject, e.g. [LSK+93], [KCK+96], by providing a sound concurrency model and an algorithm to construct it automatically from a collection of communicating labeled transition systems (LTSs). The new concurrency model, called behavior machine (BM), is an interleaved-free and finite description of concurrent and recursive behavior. The construction algorithm works as follows. First, the LTSs are mapped into a single Petri net representing the whole system. This Petri net is further used to construct its unfolding, another Petri net with a simpler structure, using an algorithm from [ERV96]. The behavior machine is then constructed from the finite prefix of a Petri net unfolding.

After the behavior machine is introduced as description model of concurrent behavior, the test derivation approach is extended to support the new model. It is shown how an extension of the transition tour can be derived from a behavior machine using algorithms already known from sequential systems. First results of the new testing approach that were obtained with a prototype implementation supporting specifications in LOTOS are discussed.

The paper is organized as follows. Section 2 introduces the model assumptions on a concurrent system. Section 3 sets up the new concurrency model. Some Petri net notions are explained in Section 4 that are needed for an easy understanding of the construction algorithm. Section 5 presents the algorithm to compute a behavior machine. In Section 6, the behavior machine serves as model for test derivation. An algorithm to derive a test suite from a behavior machine is presented. Section 7 discloses a first prototype implementation that supports LOTOS specifications, and finally, Section 8 explains concepts of the design of a distributed test architecture.

\section{A MODEL FOR DISTRIBUTED CONCURRENT SYSTEMS}

We consider distributed concurrent software systems consisting of a collection of software modules running on different host machines that are connected through a computer network. A module is implemented as a sequential unit realizing a certain 
function of the system. Modules communicate synchronously via interaction points. The synchronous communication pattern fits the properties of programming languages for concurrent systems, e.g. Ada, and function calls in high-level network programming, like remote procedure calls, which are exploited, for instance, in the middleware platform CORBA.

Starting point of our investigations is a formal specification that defines the desired behavior of the concurrent system. Sequential behavior of a module in a concurrent system is modeled as a labeled transition system (LTS). The model of an LTS is an abstraction that focuses on interactions of a module with other modules in the system and/or with its environment.

Definition 1 A labeled transition system (LTS) is defined by the quadruple $(S, A, \rightarrow$, $s_{0}$ ), where $S$ is a finite set of states; $A$ is a finite set of actions (the alphabet); $\rightarrow \subseteq S$ $\times A \times S$ is a transition relation; and $s_{0} \in S$ is the initial state.

A concurrent system $\mathfrak{I}=M_{1}\left\|M_{2}\right\| \ldots \| M_{n}$ is composed from a fixed number of communicating LTSs $M_{i}$. A composite machine $C_{\mathfrak{I}}$ of $\mathfrak{I}$ (also an LTS) is expressed by means of a composition operator $\|$ similar to that used in CSP. $P \| Q$ is the parallel composition of modules $P$ and $Q$ with synchronization of the actions common to both of their alphabets and interleaving of the others. The parallel composition $P \|$ $Q$ of two LTSs $P=\left(S_{1}, A_{1}, \rightarrow_{1}, s_{1}\right)$ and $Q=\left(S_{2}, A_{2}, \rightarrow_{2}, s_{2}\right)$ is defined as a composite LTS $(S, A, \rightarrow, s)$, where $S \subseteq S_{1} \times S_{2}, A \subseteq A_{1} \cup A_{2}, s=\left(s_{1}, s_{2}\right)$, and the transition relation $\rightarrow$ is given as follows. If $P-a \rightarrow{ }_{1} P^{\prime}$ then $(P \| Q)-a \rightarrow\left(P^{\prime} \| Q\right)$ if $a \notin A_{2}$. If $Q-a \rightarrow_{2} Q^{\prime}$ then $(P \| Q)-a \rightarrow(P \| Q)$ if $a \notin A_{1}$. If $P-a \rightarrow_{1} P^{\prime}$ and $Q-a \rightarrow_{2} Q^{\prime}$ then $(P \| Q)-a \rightarrow\left(P^{\prime} \| Q\right)$ if $a \in A_{1} \cap A_{2}$.

\section{A CONCURRENCY MODEL}

The representation of concurrent behavior in a composite machine is accomplished by a tedious repetition of concurrent actions in order to construct all possible total orders. However, concurrent actions are independent to a certain extent from their occurrence in a total order. Instead of interpreting causality information in an interleaved-based model, we apply the notion of a labeled partially ordered set and its extension to a partially ordered multiset, which are interleaved-free representations of concurrent behavior [Pra86].

Definition 2 An lposet (labeled partially ordered set) is defined by the quadruple $(E, A, \leq, l)$, where $E$ is a set of event names; $A$ is a set of action names; $\leq$ is a partial order expressing the causality information between events, i.e. $e \leq f$ if event $e$ precedes event $f$ in time; $l: E \rightarrow A$ is a labeling function assigning action names to events. Each labeled event represents an occurrence of the action labelling it, with the same action possibly having multiple occurrences. 
A pomset (partially ordered multiset) is an isomorphism class over event renaming of an lposet, denoted $[E, A, \leq, l]$. A process describing the behavior of concurrent system $\mathfrak{I}$ is a set of pomsets where each pomset describes a possible execution sequence of concurrent actions. Since the behavior of a system is frequently infinite due to recursive parts in the system description, the pomsets of a process are infinite, too. If branching occurs in a process, the set of pomsets forms an infinite pomtree [PLL+91], where an arc in the pomtree is an lposet or a concatenation of lposets, and a vertex is a branching point of the process (see Figure 3 ).

Since the construction of the composite machine from a set of communicating LTSs is not feasible in many cases due to state explosion, it follows that we need a new model that combines the advantages of both concepts: true concurrency between actions as preserved in an lposet and finiteness of the description as preserved in an LTS. This model is a behavior machine (BM), a similar model to the one introduced in [PLL+91].

Definition 3 The behavior machine of concurrent system $\mathfrak{I}$ is a quadruple $B M_{\mathfrak{I}}=$ $\left(G, L P O, T, g_{0}\right)$ consisting of a finite set of global states $G$, where each element of $G$ is an $n$-tuple of local states of all LTSs of $\mathfrak{I}$, i.e. $G \subseteq S_{1} \times \ldots \times S_{n}$; a set of finite lposets $L P O$ representing concurrent behavior in $\Im$; a concurrent transition relation $T \subseteq G \times L P O \times G$ that maps a start state to an end state when the actions of the corresponding lposet are executed; and an initial global state $g_{0}=\left(s_{1}, \ldots, s_{n}\right) \in G$.

A global state of behavior machine $B M_{\mathfrak{I}}$, excluding its initial state, expresses always a branching point or a recurrence point within concurrent system $\mathfrak{I}$. A branching point is a global state where further behavior of the system branches off. A recurrence point is a global state where the behavior of the system repeatedly continues. An lposet in $B M_{\mathfrak{S}}$ is constructed in such a way that it connects always two global states of $B M_{\mathfrak{I}}$ by a concurrent transition $t \in T$. A pomtree can be obtained from $B M_{\mathfrak{S}}$ if its concurrent transitions are unrolled. In this case, branching points in the behavior machine correspond to branching points in the pomtree, whereas recurrence points diminish. Thus, unrolling a behavior machine is similar to the construction of a spanning tree from a directed graph.

Let $t_{1}$ an $t_{2}$ be two concurrent transitions of $B M_{\mathfrak{I}}$ with $t_{1}=\left(g_{1}, l p o_{1}, g_{2}\right)$ and $t_{2}=$ $\left(g_{3}, l p o_{2}, g_{4}\right)$. The operation $t_{1} \oplus t_{2}$ expresses concatenation of the two concurrent transitions. Concatenation is allowed if $g_{2}=g_{3}$. It is carried out in such a way that each local state of the end state $g_{2}$ in $t_{1}$ is connected with the same local state of the start state $g_{3}$ in $t_{2}$. That means, the lposets $l p o_{1}$ and $l p o_{2}$ are merged according to the causal dependencies between their events.

Consider the system $\mathfrak{I}=A \| B$ whose LTSs are given in Figure 1. Under the assumption that actions $a$ and $c$ in each LTS synchronize, removal of the parallel operator by applying interleaved-based semantics rules yields the composite machine $C_{\mathfrak{g}}$. Figure 2 shows the behavior machine $B M_{\mathfrak{I}}$ of system $\mathfrak{I}$. It contains three global states $\left\{S_{0}, S_{1}, S_{2}\right\}$ and four concurrent transitions $\left\{t_{1}-t_{4}\right\}$ and describes the same behavior of system $\mathfrak{I}$ as given in Figure 1. Each concurrent transition is 
described by an lposet that exhibits concurrency among actions (see transition $t_{4}$ ). If the behavior machine is unrolled, the pomtree in Figure 3 is obtained. The process of unrolling shows the full degree of concurrency between events. For instance, if transitions $t_{2}$ and $t_{4}$ are concatenated, we realize that event $b$ is concurrent to $e$.
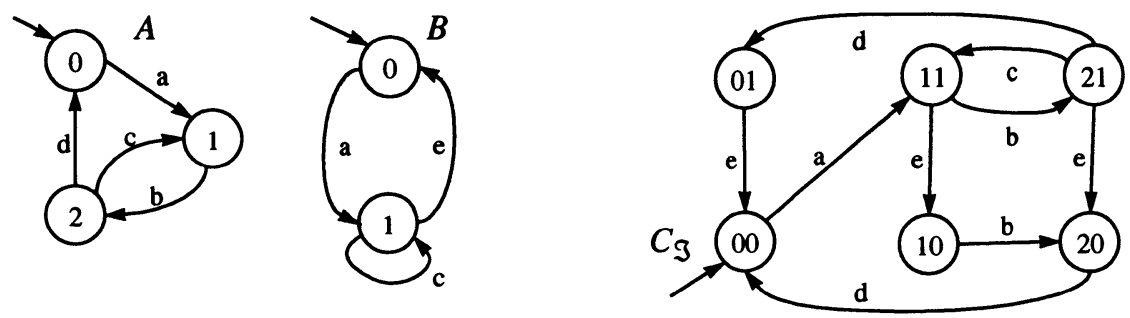

Figure 1 LTSs $A$ and $B$, and the composite machine $C_{\mathfrak{I}}$ of system $\mathfrak{I}=A \| B$.

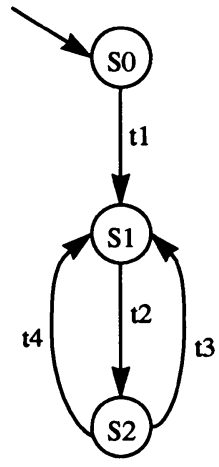

where $\mathrm{S} 0=(\mathrm{A} 0, \mathrm{~B} 0), \mathrm{S} 1=(\mathrm{A} 1, \mathrm{~B} 1), \mathrm{S} 2=(\mathrm{A} 2, \mathrm{~B} 1)$ are global states, and the concurrent transitions are

t1:
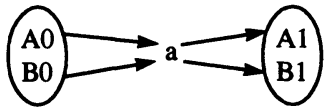

2:

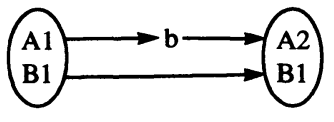

t3:

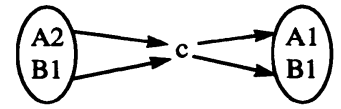

t4:

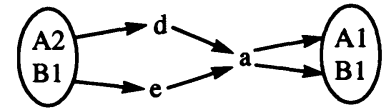

Figure 2 The behavior machine $B M_{\mathfrak{I}}$ of system $\mathfrak{I}$.

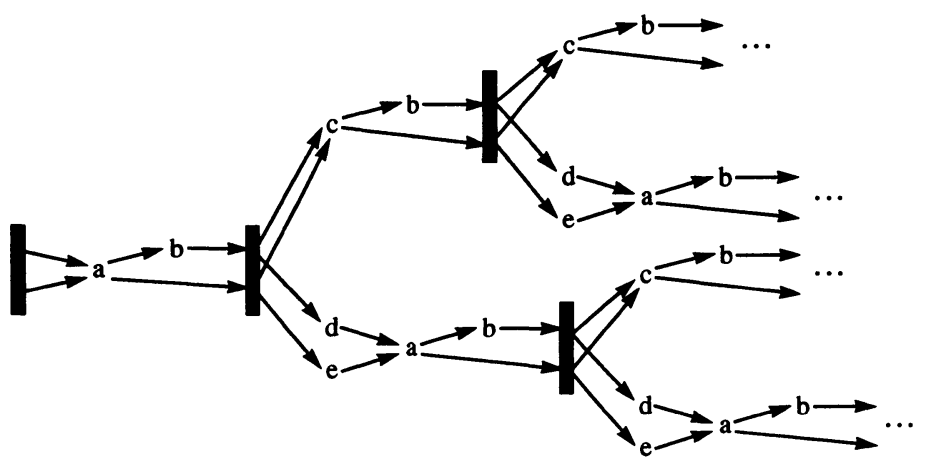

Figure 3 A pomtree of system $\mathfrak{I}=A \| B$. 
Although the behavior machine in Figure 2 is not the smallest representation of concurrent behavior due to its construction algorithm discussed below, it is still a very compact representation of concurrent behavior. In this specific example, action $a$ is redundantly represented within concurrent transitions $t_{1}$ and $t_{4}$ that can be avoided in a minimal description. Still, a behavior machine is able to distinguish concurrency from branching. This knowledge is lost in the composite machine $C_{\mathfrak{g}}$.

\section{PETRI NET CONCEPTS}

The construction algorithm of a behavior machine is based on a Petri net description of the concurrent system. In [McM95] and [ERV96], a verification approach was described that is based on the technique of net unfolding, a partial order semantics of Petri nets. The unfolding of a Petri net is another (usually infinite) net with a simpler structure. The proposed algorithms in both papers aim at constructing the initial part of the net unfolding that contains all reachable states of the original net, called the finite complete prefix.

A net is a triple $(S, T, F)$, where $S$ is the set of places, $T$ is the set of transitions, $S$ $\cap T=\varnothing, F \subseteq(S \times T) \cup(T \times S)$ is the flow relation, If $M: S \rightarrow \mathcal{N}$ is a marking of a net ( $\mathcal{N}$ denotes the set of non negative integers), the 4-tuple $N=(S, T, F, M)$ is called a Petri net [Rei91]. The unfolding of a Petri net is a (unmarked) condition-event net $(B, E, F)$, where $B$ is the set of conditions, $E$ is the set of events, with the properties: (1) it is an acyclic graph, (2) if two events (transitions) $e_{1}, e_{2} \in E$ of the unfolding are in conflict, meaning that they are enabled from the same condition (place), then there exist two paths leading to $e_{1}$ and $e_{2}$ that start at the same condition and immediately branch off from another, (3) the nodes in the unfolding have a finite number of predecessors, and (4) no event is in self-conflict.

Figure 4 depicts the Petri net description of system $\mathfrak{I}$ and the initial part of its unfolding. Note that the unfolding is not finite. For instance, if event E4 is performed, the unfolding continues by substituting place B10 with B3 and B11 with B4, respectively. This applies similarly to event E6. Events E4 and E6 are also in conflict. The branching point where the paths leading to the events branch off from another is the marking $\{$ A1, B1 $\}$, represented by the set of conditions $\{$ B5, B4 $\}$.

A local configuration $[e]$ of event $e$ in the unfolding describes a possible partially ordered run of the system which executes event $e$ as its last event. It is a set of events satisfying the following two conditions: (1) if any event is in the local configuration, then so are all of its predecessors, and (2) a local configuration is conflictfree. The local configuration captures the precedence relation between events. Any total order on these events that is consistent with the partial order is an allowed totally ordered run of the system. Throughout the paper, we use the notions local configuration and configuration interchangeable.

To compute the finite complete prefix of a Petri net, it is necessary to define a break-off condition to stop the construction of the unfolding. This is done by introducing cut-off events. An event $e$ is a cut-off event if the local configuration [e] 
belonging to event $e$ reaches a marking $\operatorname{Mark}([e])$ in the unfolding that was reached before by a smaller local configuration of a different event.

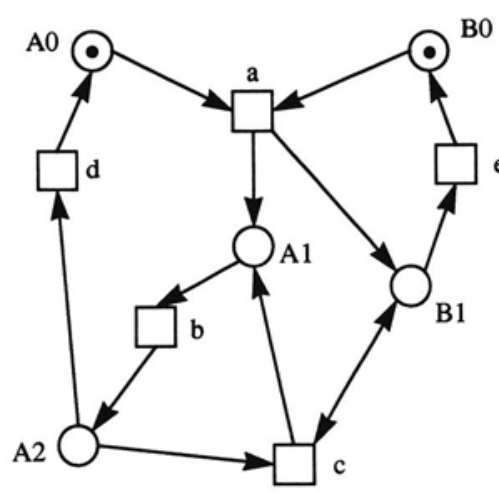

a)

B10

b)

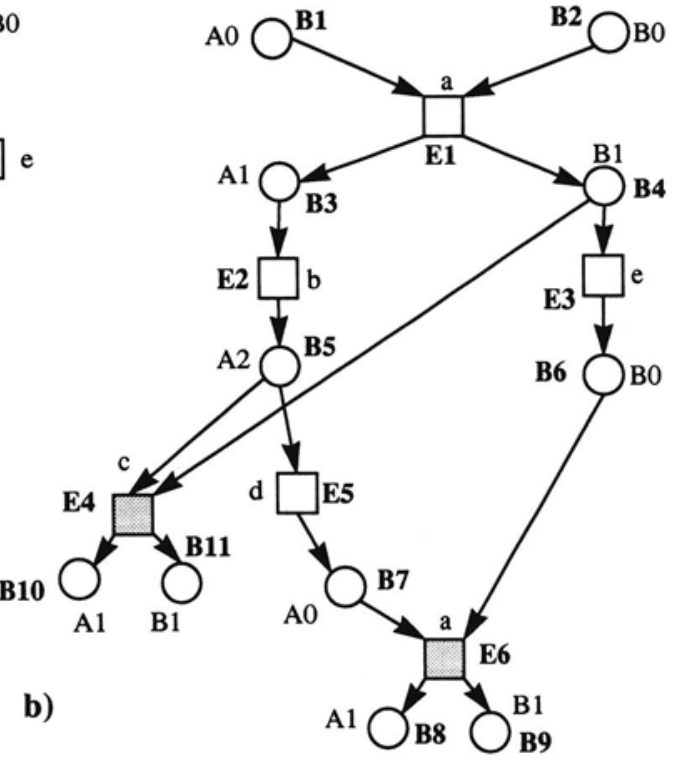

Figure 4 The marked Petri net of system $\mathfrak{I}=A \| B$ (a) and its unfolding (b).

Consider the unfolding in Figure 4b. The configuration of event E6 is the set of events $[\mathbf{E 6}]=\{\mathbf{E 1}, \mathbf{E 2}, \mathbf{E 3}, \mathbf{E 5}, \mathbf{E 6}\}$. The reachable marking of this configuration is $\operatorname{Mark}([\mathbf{E 6}])=\{\mathrm{A} 1, \mathrm{~B} 1\}$. This marking was reached before, however, by configuration $[E 1]=\{\mathbf{E} 1\}$. We say, event E6 corresponds to event E1. Since the configuration of E1 has fewer elements than the configuration of E6, it follows that E6 is a cut-off event. The second cut-off event is E4.

Proposition 1 Given the unfolding of a 1-save Petri net ${ }^{*}$. Any deadlock-free system is completely represented by the finite set of tuples of cut-off and corresponding events $\left\{\left(e_{\text {cutoff }_{1}}, e_{\text {corresp }_{1}}\right),\left(e_{\text {cutoff }_{2}}, e_{\text {corresp }_{2}}\right), \ldots,\left(e_{\text {cutoff }_{n}}, e_{\text {corresp }_{n}}\right)\right\}$, where the two configurations of events in a tuple reach the same marking, $\left.\operatorname{Mark}^{2}\left(e_{\text {cutoff }_{i}}\right]\right)=$ $\operatorname{Mark}\left(\left[e_{\text {corresp }_{i}}\right]\right)$.

The proposition requires cut-off events for each execution branch in the net unfolding. Their existence was proven in [ERV96]. A deadlocking system, however, reaches a final marking that does not relate to a cut-off event. The construction algorithm of a behavior machine is currently restricted to concurrent systems without

\footnotetext{
* A 1-save Petri net is a net whose places contain at most one token at a certain time. This type of net is obtained, for instance, if the net is constructed from a set of communicating LTSs.
} 
deadlocks. This is, however, not a restriction for the purpose of test derivation since we usually require that the specification of a system has been verified to be deadlock-free before it can be implemented.

\section{CONSTRUCTION ALGORITHM OF A BEHAVIOR MACHINE}

The first step in constructing a behavior machine from a set of communicating LTS is a transformation of the LTSs into a global Petri net. After the transformation, the unfolding algorithm is applied to unfold the Petri net and to construct the set of pairs of cut-off and corresponding events. Finally, the behavior machine is constructed from the unfolding.

Constructing a Petri net from a set of communicating LTSs is simple. The following algorithm is applied: first, each single LTS is transformed into a Petri net; then, all Petri nets are merged according to the synchronization constraints in order to obtain a global Petri net. If the same action name labels several transitions in several LTSs, then a net transition for each allowed way of synchronization has to be constructed in the global Petri net. The transformation was already presented in [GaSi90] and is used in the Casar/Aldebaran toolset that supports verification of specifications given in the formal description language LOTOS.

Figure 4a shows the Petri net constructed from the two LTSs in Figure 1. The next step is the construction of the finite complete prefix. This is done by applying the algorithm presented in [ERV96]. Figure 4b depicts the prefix of the example system. The last step, the construction of the behavior machine, is described below.

We assume that the finite complete prefix of a Petri net unfolding, including the set of cut-off and corresponding events, is given. The local configuration of an event describes an execution path through the behavior machine from the initial state to this particular event. The marking reached by the configuration of an event defines a global state in the behavior of a concurrent system. The reachable marking of a configuration can be identified with places in the unfolding that are reached if all events in the configuration are executed.

When the behavior machine is constructed, it is not necessary to compute all reachable markings in the unfolding. Instead, only those reachable markings have to be known that are recurrence or branching points. Cut-off events and events corresponding to them define recurrence points of the behavior machine. Yet, branching points have to be computed.

To identify the branching points, we do the following considerations. Given the finite complete prefix of an unfolding, each local configuration of a cut-off event or a corresponding event starts in the initial state of the system, i.e. the initial marking, and ends in a marking reached by the configurations of those events. Since the finite complete prefix covers all reachable states of the system, branching points exist only somewhere inside the configurations of cut-off and corresponding events. If we analyze any two configurations $\left[e_{1}\right]$ and $\left[e_{2}\right]$ with $e_{1} \neq e_{2}$ from the same unfolding, we realize that the configurations start with a same subset of events and branch off 
from another after a certain event $e_{\text {branch }}$ occurred in both configurations. Now, a branching point can be defined exactly by the reachable marking of the configuration formed by this event $e_{\text {branch }}$ assuming that $e_{\text {branch }} \in\left[e_{i}\right]$, and $\left[e_{\text {branch }}\right]$ is the maximal configuration that holds the condition $\left[e_{\text {branch }}\right] \subseteq\left[e_{i}\right]$, with $i=\{1,2\}$.

This observation leads to the construction algorithm. It takes the set of cut-off events and corresponding events that are contained in the finite complete prefix of an unfolding as input. The idea behind the algorithm is to construct the configurations of the given cut-off and corresponding events first. Then, the events in the configurations are analyzed in order to identify the branching points.

- let $E$ be the set of cut-off and corresponding events in a finite prefix;

$2 \cdot$ let $\mathcal{E}$ initially be the set of configurations from all events in $E$, i.e.

$$
\mathcal{E}=\left\{\left[e_{1}\right],\left[e_{2}\right], \ldots\right\} \text {; }
$$

3 forall configurations $[e] \in \mathcal{E}$ do

forall events $d \in[e]$ with $d \neq e$ do

forall configurations $[d] \in \mathcal{E}$ do

Figure 5 Generation of configurations represented in the behavior machine.

As discussed above, a branching point is defined by the reachable marking of a maximum configuration contained within two or more other configurations. To identify these points, we analyze the successor places of an event $e$. If at least one of the successor places has more than one successor event, the reachable marking of the configuration $[e]$ might be a branching point in the behavior machine (static conflict). Since this result is obtained from a local analysis of a single event rather than from an analysis of the global system, not all events found in this way refer 
really to a branching point. The algorithm in Figure 5 takes into account this aspect and returns only those events and their configurations that will be finally considered in the construction of a behavior machine.

The initial set of configurations $\mathcal{E}$ is obtained from the configurations of cut-off and corresponding events contained in the prefix of the unfolding (line 2 in Figure 5). In the next step, further configurations of events are added to $\mathcal{E}$ if these events possess successor places that cause local branching (lines 3-10). The thi:d step (lines 11-16) determines whether a configuration is contained in another ore and marks the maximal configuration that fulfills this property. The final step (lines 17-20) deletes configurations of events added to $\mathcal{E}$ before if they are not marked as maximal configurations or if they are marked only once in another configuration. That means, configurations that do not determine a branch in the behavior are omitted in the construction of the behavior machine.

$1-$ let $\mathcal{E}$ be the set of local configurations;

2 global_states $=\varnothing$;

3 forall configurations $[d] \in \mathcal{E}$ do

4 - compute the reachable marking reachable_marks of $[d]$;

5

6 end global_states $=$ global_states $\cup\{$ reachable_marks $\}$;

7 conc trans $=\varnothing$;

8 forall configurations $[d] \in \mathcal{E}$ do

$9 \quad$ let $[e]$ be the maximal configuration of $[d]$;

10 conc_trans $=$ conc_trans $\cup\{[d] \backslash[e]\}$;

11 end

12 return global_states, conc_trans;

Figure 6 Construction of global states and concurrent transitions in a BM.

The algorithm in Figure 5 returns the set of configurations $\mathcal{E}$ relevant in the construction of the behavior machine, i.e., the configurations have the property that they reach the marking of a recurrence point or a branching point. In the next algorithm (Figure 6), this knowledge is used to construct the global states and the concurrent transitions of the behavior machine of a concurrent system.

In line 4 of Figure 6 , the reachable marking is computed. Note that the reachable markings are the same for the configuration of a cut-off event and the configuration of its corresponding event, thus the second computation is redundant. A concurrent transition in a behavior machine is computed in line 10. It is simply the set difference of configuration $[d]$ and the maximal configuration $[e]$ contained in $[d]$. This computation is correct since the configuration $[e]$ is a subset of $[d]$, and all events in $[e]$ occur in the behavior machine in one or more other concurrent transitions. The behavior machine is now nearly complete. The missing initial global state of the behavior machine is computed from the reachable marking of the empty configuration, i.e., it is the initial marking in the Petri net. 
The construction of a behavior machine is based on cut-off events and corresponding events in the finite complete prefix and the configurations belonging to them. If we assume that the events and conditions of the prefix are stored in doubly linked lists, local configurations and reachable markings can be computed in linear time. Thus, the highest computational complexity of the construction algorithm is contained in the identification of branching points (Figure 5, lines 11-16). The complexity of this algorithm is bound on $O\left(n^{2} \cdot\left(\log _{k} n\right)^{2}\right)$, where $n$ is the number of places in the prefix of the unfolding, and $k$ is the largest number of successor places of any transition. All other parts of the construction algorithm are less complex.

Table 1 Results of the dining philosophers example, computed on a Sparc Station 5.

\begin{tabular}{llllll}
\hline $\begin{array}{l}\text { \# } \\
\text { philo. }\end{array}$ & $\begin{array}{l}\text { \# reachable } \\
\text { states }\end{array}$ & $\begin{array}{l}\text { \# global } \\
\text { states }\end{array}$ & $\begin{array}{l}\text { \#concurrent } \\
\text { transitions }\end{array}$ & $\begin{array}{l}\text { memory } \\
\text { (kByte) }\end{array}$ & $\begin{array}{l}\text { computation } \\
\text { time (sec) }\end{array}$ \\
\hline 5 & 392 & 16 & 35 & 36 & 0.11 \\
7 & 4,247 & 36 & 77 & 61 & 0.73 \\
9 & 46,763 & 64 & 135 & 98 & 2.96 \\
11 & 510,116 & 100 & 209 & 151 & 9.12 \\
13 & $5,564,522$ & 144 & 299 & 222 & 22.76 \\
15 & - & 196 & 405 & 314 & 48.83 \\
\hline
\end{tabular}

To demonstrate the feasibility of the construction algorithm, we compute the behavior machines for a variable number of processes of the dining philosophers example. The results are given in Table 1 for a varying number of philosophers. Note that this example contains a deadlock state whose configuration is not represented in the behavior machine due to Proposition (1). However, all other behavior parts are truly represented. The second column shows the number of reachable states computed by a traditional reachability analysis. The number of states grows clearly exponentially with the number of philosophers. The following two columns reveal the numbers of global states and concurrent transitions of the constructed behavior machines. We realize that the number of global states increases slightly worse than quadratic. Even though the computation time increases fast and seems to be a function of $n^{5.5}$, where $n$ is the number of philosophers, the time is still reasonable small. This is also particularly true for the memory space used. The computation time of the finite complete prefix that is used as input for our construction algorithm was always less or around few seconds.

\section{TEST GENERATION BASED ON BEHAVIOR MACHINES}

The behavior machine is an appropriate model for test suite derivation. A test suite has to fulfill certain properties to be useful in software testing. Especially, it must 
distinguish faulty implementations from correct ones according to a chosen conformance relation. A conformance relation commonly used in testing is the trace equivalence between LTSs modeling the specification and the implementation. We extend trace equivalence over LTSs to an equivalence over behavior machines. First, equivalence of lposets is defined as follows borrowing ideas from [Bri88].

Definition 4 Lposet $l p o_{1}=\left(E_{1}, A_{1}, \leq_{1}, l_{1}\right)$ reduces lposet $l p o_{2}=\left(E_{2}, A_{2}, \leq_{2}, l_{2}\right)$, denoted $l p o_{1} \sim l p o_{2}$, iff $A_{1} \subseteq A_{2}$, and for all $e, f \in E_{1}$, if $e \leq_{1} f$ then there exist $r, s \in$ $E_{2}$ such that $r \leq_{2} s$, and $l_{2}(r)=l_{1}(e), l_{2}(s)=l_{1}(f)$. Two lposets are equivalent, lpo $o_{1} \approx$ $l p o_{2}$, iff $l p o_{1} \sim l p o_{2}$ and $l p o_{2} \sim l p o_{1}$.

We define further a sequence seq of lposets as a concatenation of a matching sequence of lposets according to the $\oplus$ operator (see Section 3): $s e q=l p o_{1} \oplus l p o_{2}$ $\oplus \mathrm{lpo}_{3} \oplus \ldots$. Thus, a sequence of lposets describes a pomset, i.e. an execution branch of the concurrent system as depicted in its pomtree. Two sequences $s e q_{1}$ and $s e q_{2}$ equal, $s e q_{1}=s e q_{2}$, if their lposets are equivalent.

Definition 5 Given two concurrent systems $\mathfrak{I}$ and $\Re$ and their behavior machines $B M_{\mathfrak{S}}$ and $B M_{\Re}$, respectively. Let $\operatorname{Se}(B M)$ refer to the set of sequences of lposets of which behavior machine $B M$ is able to perform. $\mathfrak{I}$ and $\Re$ are equivalent, $\mathfrak{I} \approx_{\mathrm{c}} \Re$, iff $\operatorname{Seq}\left(B M_{\mathfrak{S}}\right)=\operatorname{Seq}\left(B M_{\Re}\right)$.

A test suite consisting of a finite set of finite test cases is sound w.r.t. a fault model if any conforming implementation passes the test suite. A test suite is complete w.r.t. a fault model if any non-conforming implementation from the implementation domain fails the test suite [PBY96]. An implementation domain that is often considered in conformance testing is the set of implementations with acceptance faults, i.e., these implementations may not accept all actions by corresponding transitions as required in the specification, thus they reduce the specification [Lan90].

Acceptance faults can be detected by a transition cover of the concurrent system. A transition cover is usually defined over an LTS as a set of traces covering all transitions in the LTS. We extend now the notion of a transition tour [ADL+91] over a behavior machine as the least sequence of lposets covering all lposets. Such a sequence of lposets can be seen as a "transition cover" of a behavior machine. The extended notion is called concurrent transition tour (CTT) [UlCh95].

Definition 6 (CTT) A concurrent transition tour through a behavior machine $B M_{\mathfrak{S}}$ of a concurrent system $\mathfrak{I}$ is the least pomset $C T T=\left[E_{C T T}, A_{C T T}, \leq, l\right]$ such that all actions of $\mathfrak{I}$ are covered in the pomset, i.e. if $a \in A_{1} \cup A_{2} \cup \ldots \cup A_{n}$, then $a \in$ $A_{C T T}$, and $E_{C T T}$ is minimal.

Derivation of a CTT from a behavior machine is straightforward. Since the description of concurrent behavior is reduced to a finite directed graph, simple graph algorithms can be applied. To construct a CTT, an algorithm that solves the Chinese 
postman problem is appropriate [ADL+91]. First, all strongly-connected components of maximum size contained in a behavior machine are computed. After that, a CTT is derived for each strongly-connected component. This approach assures full coverage of all transitions in the behavior machine. The complete algorithm is given in Figure 7. Note however that the size of CTTs computed in this algorithm might not be minimal if we assume, for example, a behavior machine consisting of two components where the second component is reachable through the first one. Further optimization strategies might become applicable in this case.

1 Find all strongly-connected subgraphs of maximum size $b m_{1}^{s}, \ldots, b m_{n}^{s}$ in behavior machine $B M_{\mathfrak{I}}$.

2 For each $b m^{s}{ }_{i}$ find the shortest path $p_{i}$ from the initial state of $b m$ to $b m^{s}{ }_{i}$.

3 For each $\mathrm{bm}^{s}{ }_{i}$ find the Chinese postman tour $p t_{i}$.

4 A CTT of a subgraph of $b m$ is found by concatenation of $p_{i}$ and $p t_{i}$ : $C T T_{i}=p_{i} \oplus p t_{i}$.

5 The test suite is the set of all CTTs found: $T S=\left\{C T T_{1}, \ldots, C T T_{n}\right\}$.

Figure 7 Test suite derivation.

Consider the behavior machine $B M_{\mathfrak{I}}$ of the system $\mathfrak{I}=A \| B$ in Figure 2. It contains one strongly-connected component consisting of the states $S_{1}$ and $S_{2}$. The initial path to reach this component is given by concurrent transition $t_{1}$. The Chinese postman tour through the component is the sequence of concurrent transitions $t_{2} \oplus t_{3} \oplus$ $t_{2} \oplus t_{4}$. The final test suite of system $\mathfrak{I}$ contains only a single CTT and is given in Figure 8 as a time-event sequence diagram where the gray-shaped arrows denote synchronization constraints between the modules $A$ and $B$. This test suite describes the shortest path through the concurrent system fulfilling the requirements of a CTT.

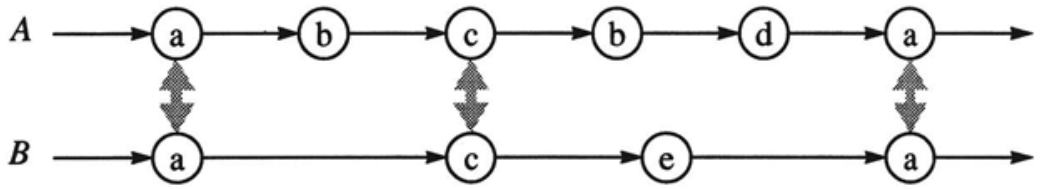

Figure 8 A concurrent transition tour for concurrent system $\mathfrak{I}=A \| B$.

\section{A PROTOTYPE IMPLEMENTATION}

The algorithms presented in Section 5 and Section 6 have been implemented as a first prototype tool to support concurrent test suite derivation from LOTOS specifications. In order to operate with Full LOTOS, we use the Casar/Aldebaran verification toolset [GaSi90]. Caesar produces the Petri net of a LOTOS specification as it is the required input for the construction of a behavior machine. The produced Petri 
net has the advantage that values of variables are still represented symbolically. Thus, the Petri net description of the concurrent system remains small in size.

The Petri net of the specification serves then as input to the construction of the complete finite prefix of the net using the $P E P$ tool [GrBe96]. Currently however, we do not support symbolic evaluation of data terms in LOTOS predicates or guard expressions. The behavior is considered to be executable under all cases instead. Consequently, the constructed prefix of the Petri net unfolding contains dead execution branches that cannot be executed by a correct implementation. At the moment, this code has to be removed manually in order to generate only executable test suites. Eventually, test suites according to the acceptance fault model are generated from the behavior machine.

A further drawback of the current prototype is the use of the verification tool $P E P$. Since $P E P$ stops to unfold an execution branch of the Petri net if it has covered all reachable states (a sufficient condition in verification) instead of continuing to unfold until a cut-off event is reached, the resulting behavior machine contains uncompleted cycles. A new implementation of the unfolding algorithm is therefore necessary. If this work will be carried out, the construction of a behavior machine should then be integrated into a single tool.

\section{DISTRIBUTED TESTER DESIGN}

Although the derivation of test suites from concurrent systems appears to be manageable now by the approach presented before, there is still the problem to apply a concurrent test suite in a real test environment. Here we are faced with some unpleasant properties of concurrent systems, namely with the unpredictable progress in the concurrent modules when executing a test run and with hidden communication between modules that remain unobserved by the tester. Both issues result in nondeterministic behavior of the implementation under test (IUT). Whereas the second issue can be solved by applying a gray-box testing approach, the first one requires special means to ensure a deterministic test run, e.g. the instant replay technique presented in [TCO91].

In the simplest case of a test architecture, the tester is represented as a single module that implements a CTT of a given test suite. Since a tester module can exhibit only sequential behavior, the concurrent behavior of a CTT must be linearized in such a way that the causal relationships between events remain unchanged. For example, the CTT in Figure 8 can be implemented as the sequence of events "a.b.c.b.d.e.a" that fulfills the requirements. If the IUT is correctly implemented according to the chosen fault model, then it must be able to accept any sequence of events represented in the CTT and the selection of the actual sequence used in testing is arbitrary.

In case that the tester consists itself of distributed concurrent tester parts, further efforts are required to obtain reliable test results after a test run. Here, each tester part observes a sub-set of events of the IUT. The partial behavior containing those 
events visible to a tester part can be obtained from a projection from the complete CTT to a partial CTT containing only the visible events. Each tester part will then execute the behavior of the projected CTT. Yet, means are required to obtain a globally ordered test run of the IUT from the observed partial behavior, e.g. synchronization messages between tester parts.

\section{CONCLUSIONS}

The model of a behavior machine is used to support automatic test derivation for concurrent systems. The model has its merits as a finite description of concurrent behavior that still exhibits true concurrency among actions. Furthermore, a behavior machine distinguishes concurrency from execution branching. Vertices in the behavior machine refer usually to a small subset of the set of reachable global states in the system. The main contributions of this paper are the presentation of an algorithm that constructs a behavior machine from a Petri net unfolding as well as the application of the behavior machine to the realm of test derivation. For the purpose of test derivation, the notion of trace equivalence was extended to cope with concurrent behavior.

The presented approach to construct a behavior machine can be improved. Especially, the Petri net unfolding algorithm and the construction algorithm of the behavior machine should be combined into a single tool. Other extensions of a behavior machine may include the support of inputs and outputs and the supply of operations over behavior machines, e.g. the composition of two behavior machines, projections of behavior machines to submachines and other operations. Last but not least, more expanded fault models should be investigated and optimized test derivation algorithms should be elaborated for them.

Currently, other approaches are under investigation to construct a concurrency model that avoid the use of Petri nets [Hen97], though the applicability of the presented algorithms, i.e. their computational complexity, has still to be determined.

An extended version of the paper and other related work can be found on the web page http://irb.cs.uni-magdeburg.de/ ulrich/.

Acknowledgment The authors wish to thank Alex Petrenko for a very fruitful discussion and for his hints that helped to improve the quality of the paper.

\section{REFERENCES}

[ADL+91] A. V. Aho, A. T. Dahbura, D. Lee, M. Ü. Uyar: An optimization technique for protocol conformance test generation based on UIO sequences and rural Chinese postman tours; IEEE Transactions on Communications, vol. 39, no. 11 (Nov. 1991); pp. 1604-1615.

[Bri88] Ed Brinksma: A theory for the derivation of tests; 8th Int'1 Symposium 
on Protocol Specification, Testing and Verification (PSTV'88), Atlantic City, USA; 1988.

[ERV96] J. Esparza, S. Römer, W. Vogler: An improvement of McMillan's unfolding algorithm; 2nd Int'l Workshop on Tools and Algorithms for the Construction and Analysis of Systems; Passau, Germany; 1996.

[Fer96] J.-C. Fernandez, C. Jard, Th. Jéron, César Viho: Using on-the-fly verification techniques for the generation of test suites; 8th Int'l Conference on Computer Aided Verification (CAV'96); New Brunswick, New Jersy, USA; 1996.

[GaSi90] H. Garavel, J. Sifakis: Compilation and verification of Lotos specifications; 10th Int'l Symposium on Protocol Specification, Testing and Verification (PSTV'90); Ottawa, Canada; 1990; pp. 379-394.

[GrBe96] B. Grahlmann, E. Best: PEP - More than a Petri net tool; 2nd Int'1 Workshop on Tools and Algorithms for the Construction and Analysis of Systems (TACAS'96); Passau, Germany; 1996.

[Hen97] O. Henniger: On test case generation from asynchronously communicating state machines; 10th Int'1 Workshop on Testing of Communicating Systems (IWTCS'97); Cheju Island, Korea; Sep. 1997.

[KCK+96] M. C. Kim, S. T. Chanson, S. W. Kang, J. W. Shin: An approach for testing asynchronous communicating systems; 9th Int'1 Workshop on Testing of Communicating Systems; Darmstadt, Germany; Sep. 1996.

[Lan90] R. Langerak: A testing theory for LOTOS using deadlock detection; 9th Int'1 Symposium on Protocol Specification, Testing and Verification (PSTV'90); Enschede, The Netherlands; 1990.

[LSK+93] D. Lee, K. K. Sabnani, D. M. Kristol, S. Paul: Conformance testing of protocols specified as communicating FSMs; IEEE INFOCOM'93; San Fransisco, CA, USA; 1993.

[McM95] K. L. McMillan: A technique of state space search based on unfolding; Formal Methods in System Design, vol. 6, no. 1 (Jan. '95); pp. 45-65.

[Pra86] V. Pratt: Modelling Concurrency with partial orders; International Journal of Parallel Programming, vol. 15, no. 1 (Feb. 1986); pp. 33-71.

[PLL+91] D. K. Probst, H. F. Li, K. G. Larsen, A. Skou: Partial-order model checking: a guide for the perplexed; 3nd Int'l Conference on Computer-aided Verification (CAV'91); Aalborg, Denmark; 1991.

[PBY96] A. Petrenko, G. v. Bochmann, M. Yao: On fault coverage of tests for finite state specifications; Special Issue on Protocol Testing, Computer Networks and ISDN Systems, vol. 29, 1996; pp. 81-106.

[Rei91] W. Reisig: Petri nets; Springer Verlag, 1991.

[TCO91] K. C. Tai, R. H. Carver, E. E. Obaid: Debugging concurrent Ada programs by deterministic execution; IEEE Transactions on Software Engineering, vol. 17, no. 1 (Jan. 1991); pp. 45-63.

[U1Ch95] A. Ulrich, S. T. Chanson: An approach to testing distributed software systems; 15th Int'1 Symposium on Protocol Specification, Testing and Verification (PSTV'95); Warsaw, Poland; pp. 107-122; 1995. 